\title{
Insulin-Sensitizing Effect of LXR Agonist T0901317 in High-Fat Fed Rats is Associated with Restored Muscle GLUT4 Expression and Insulin-Stimulated AS160 Phosphorylation
}

\author{
Marcin Baranowskia Piotr Zabielskia Agnieszka U. Błachnio-Zabielska ${ }^{a}$ \\ Ewa Harasima ${ }^{a}$ Adrian Chabowski ${ }^{a}$ Jan Górskia \\ aDepartment of Physiology, Medical University of Białystok, Białystok, Poland
}

\section{Key Words}

Akt/PKB • Insulin resistance $\cdot$ IRS1 $\bullet$ Lipid mediators $•$ Skeletal muscle

\begin{abstract}
Background/Aim: Liver X receptors (LXRs) are ligand-activated transcription factors that were shown to stimulate hepatic lipogenesis leading to liver steatosis and hypertriglyceridemia. Despite their pro-lipogenic action, LXR activators normalize glycemia and improve insulin sensitivity in rodent models of type 2 diabetes. Antidiabetic action of LXR agonists is thought to result from suppression of hepatic gluconeogenesis. However, it remains unclear whether LXR activation affects muscle insulin sensitivity. In the present study we attempted to answer this question. Methods: The experiments were performed on male Wistar rats fed for 5 weeks on either standard chow or high fat diet. The latter group was further divided into two subgroups receiving either selective LXR agonist - T0901317 (10mg/kg/d) or vehicle during the last week of the experiment. All animals were then anaesthetized and samples of the soleus as well as red and white sections of the gastrocnemius muscle were excised. Results: As expected, administration of T0901317 to high-fat fed rats augmented diet-induced hyperlipidemia. Nevertheless, it also normalized glucose tolerance and improved insulin-stimulated glucose uptake in isolated soleus muscle. In addition, LXR agonist completely restored glucose transporter 4 expression and insulin-stimulated Akt substrate of $160 \mathrm{kDa}$ phosphorylation in all investigated muscles. Insulin-sensitizing effect of T0901317 was not related to changes in intramuscular level of lipid mediators of insulin resistance, since neither diacylglycerols nor ceramide content was affected by the treatment. Conclusion: We conclude that improvement in muscle insulin sensitivity is one of the mechanisms underlying the antidiabetic action of LXR activators.
\end{abstract}




\section{Introduction}

Liver X receptors (LXRs) are ligand-activated transcription factors of the nuclear hormone receptor superfamily. There are two known LXR isoforms termed $\alpha$ and $\beta$ that are highly related and share $\sim 78 \%$ identity of their amino acid sequences in DNA and ligand-binding domains [1]. Whereas LXR $\beta$ is broadly expressed, high expression of LXR $\alpha$ is restricted to certain tissues such as liver, adipose tissue, intestine, kidney and lung [2]. Ligand-activated LXRs form heterodimers with the obligate partner 9-cis retinoid acid receptor and promote transcriptional activation by binding to a specific DNA sequence (LXR response element) in the promoter region of the target genes [3]. Endogenous LXR agonists include a number of oxidized cholesterol derivatives such us 22(R)-hydroxycholesterol, 20(S)-hydroxycholesterol, 24(S)-hydroxycholesterol and 24(S),25-epoxycholesterol [4]. In addition, some synthetic non-steroidal LXR activators have been developed. Among them, T0901317 and GW3965 are the most commonly used in experimental studies. Both are potent dual $L X R \alpha / \beta$ agonists [5].

LXRs have been proposed to operate as intracellular sterol sensors protecting the cells from cholesterol overload. When the amount of cholesterol increases there is a subsequent accumulation of cellular oxysterols which, in turn, activate LXRs to enhance transcription of a whole array of genes involved in reverse cholesterol transport and its conversion to bile acids in the liver [2]. This finding led to identification of synthetic LXR agonists as potent antiatherogenic agents in murine models of atherosclerosis [6, 7]. In addition, synthetic (but not endogenous) LXR agonists were found to stimulate hepatic lipogenesis via upregulation of sterol regulatory element binding protein (SREBP) 1c which leads to severe liver steatosis, hepatomegaly and hypertriglyceridemia [8].

In addition to their effects on cholesterol and fatty acid metabolism, synthetic LXR agonists have been found to possess potent antidiabetic properties. T0901317 and GW3965 were reported to normalize glycemia and improve whole body insulin sensitivity in animal models of diet-induced insulin resistance and type 2 diabetes. This action of LXR agonists is thought to result from downregulated expression of hepatic gluconeogenic genes, however, there is some data indicating that they also improve peripheral insulin sensitivity [2]. Although LXRs are expressed in human and rodent skeletal muscle $[9,10]$, there is very few information on the effect of LXR activation on muscle insulin sensitivity, and most of the available studies were performed on cultured myotubes. Therefore, we aimed to determine whether amelioration of muscle insulin resistance contributes to the insulin-sensitizing effect of T0901317 in high-fat fed rats.

\section{Materials and Methods}

Animals and study design

The investigation conforms with the "European Convention for the Protection of Vertebrate Animals used for Experimental and other Scientific Purposes" (Council of Europe No 123, Strasbourg 1985) and was approved by the Ethical Committee for Animal Experiments at the Medical University of Białystok. Male Wistar rats (80-100g) were housed under controlled conditions $\left(21^{\circ} \mathrm{C} \pm 1,12 \mathrm{~h}\right.$ light/12h dark cycle, light on at 06:00h) with unlimited access to water. The animals were fed ad libitum for 5 weeks on either standard chow (Agropol, Motycz, Poland, 2.8 gm\% of fat) or high fat diet (D12492, Research Diets Inc., $35 \mathrm{gm} \%$ of fat). The latter group was further divided into two subgroups receiving either vehicle or dual LXR $\alpha / \beta$ agonist T0901317 (Cayman Chemicals, $10 \mathrm{mg} / \mathrm{kg} / \mathrm{d}$, suspended in $0.5 \%$ carboxymethylcellulose) throughout the last week of the experiment. The solutions were administrated once daily by an oral gavage. On the last day of the experiment all rats were anaesthetized by intraperitoneal injection of pentobarbital in the dose of $80 \mathrm{mg} / \mathrm{kg}$ of body weight. Samples of the soleus as well as red (RG) and white (WG) sections of the gastrocnemius muscle were excised and immediately freeze-clamped with aluminum tongs precooled in liquid nitrogen. Blood taken from the abdominal aorta was collected in heparinized tubes, centrifuged, the plasma separated and flash-frozen in liquid nitrogen. All samples were stored at $-80^{\circ} \mathrm{C}$ until analysis. 


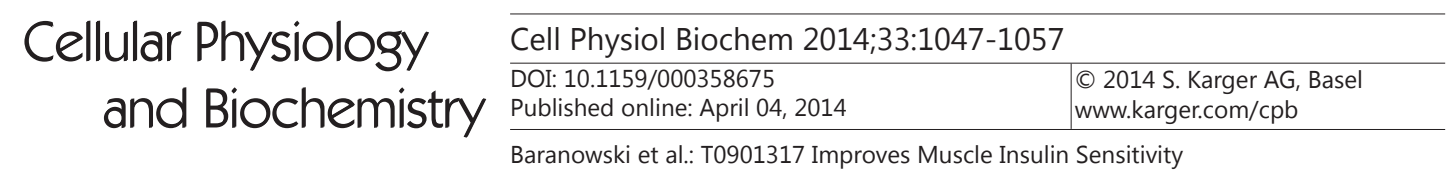

Glucose tolerance test and insulin tolerance test

For glucose tolerance test rats were fasted overnight for $12 \mathrm{~h}$ and then gavaged with glucose $(2.5$ $\mathrm{g} / \mathrm{kg}$ of body weight). Blood glucose in samples obtained from a tail nick was measured with HemoCue 201+ analyzer (HemoCue AB, Sweden) at 0, 15, 30, 60 and 120 minutes after the glucose load. For insulin tolerance test rats were fasted as described above and then injected intraperitoneally with regular human insulin (Humulin R, Eli Lilly, $0.75 \mathrm{U} / \mathrm{kg}$ of body weight). Blood glucose concentration was then determined at $0,15,30,45,60,90$ and 120 minutes after the injection.

\section{Acute insulin stimulation in vivo}

Rats were fasted overnight for $12 \mathrm{~h}$ and then anaesthetized with pentobarbital. Muscle samples were taken from one hind limb and immediately frozen in liquid nitrogen. A bolus of insulin $(10 \mathrm{U} / \mathrm{kg}$ of body weight) was then injected into the tail vein. After $4 \mathrm{~min}$ muscle samples were rapidly excised from the opposite limb and immediately frozen in liquid nitrogen.

Glucose uptake in incubated soleus muscle strips

Longitudinal soleus muscle strips were fixed in vertical position at resting length and preincubated in glass vials containing Krebs-Henseleit buffer at pH 7.4, supplemented with $5.5 \mathrm{mM}$ glucose, $2 \mathrm{mM}$ sodium pyruvate and $1.5 \%$ defatted bovine serum albumin (BSA) for $30 \mathrm{~min}$ at $30^{\circ} \mathrm{C}$, with gentle agitation $(110 /$ min), under continuous gassing with $95 \% \mathrm{O}_{2} / 5 \% \mathrm{CO}_{2}$. Temperature, shaking and gassing remained constant throughout subsequent incubation.

2-deoxy-D-glucose transport was measured using a modified method described by Hansen et al. [11]. Glucose uptake was determined in soleus muscle strips during 1) basal conditions and 2) insulin stimulation. Briefly, after pre-equilibration, muscle strips were washed and glucose transport was determined in palmitate-free Krebs-Henseleit buffer $(2 \mathrm{ml})$ supplemented with $0.5 \mu \mathrm{Ci} / \mathrm{ml}\left[{ }^{3} \mathrm{H}\right]$-2-deoxy-D-glucose, 0.2 $\mu \mathrm{Ci} / \mathrm{ml}\left[{ }^{14} \mathrm{C}\right]$-mannitol, $8 \mathrm{mM}$ 2-deoxy-glucose, $28 \mathrm{mM}$ mannitol and $4 \mathrm{mM}$ sodium pyruvate in the presence or absence of insulin for $20 \mathrm{~min}$. Thereafter, muscles were blotted of excess fluid on filter paper, weighed and frozen in liquid nitrogen. Muscle strips were digested by boiling in $300 \mu \mathrm{l}$ of $1 \mathrm{M} \mathrm{NaOH}$ at $80^{\circ} \mathrm{C}$ for 10 $\mathrm{min}$. Digested muscles were then neutralized with $300 \mu \mathrm{l}$ of $1 \mathrm{M} \mathrm{HCl}$. Radioactivity of muscle extracts was determined in duplicate by liquid scintillation counting (for dual labels ${ }^{3} \mathrm{H}$ and ${ }^{14} \mathrm{C}$ ), and the extracellular and intracellular spaces were calculated. Glucose uptake $\left(\mu \mathrm{mol}\left[{ }^{3} \mathrm{H}\right]-2\right.$-deoxy-D-glucose/g) data was calculated from intracellular $\left[{ }^{3} \mathrm{H}\right]$-2-deoxy-D-glucose accumulation after correcting for interstitial volume with $\left[{ }^{14} \mathrm{C}\right]-$ mannitol.

\section{Plasma measurements}

Concentrations of plasma glucose, triacylglycerols, nonesterified fatty acids (NEFA) and insulin were determined with the use of Glucose Oxidase Reagent Set (Pointe Scientific Inc.), Serum Triacylglycerol Determination Kit (Sigma), Wako NEFA C kit (Wako Chemicals) and Rat Insulin ELISA kit (Mercodia), respectively.

\section{Muscle diacylglycerols and ceramide}

Samples were pulverized in an aluminum mortar precooled in liquid nitrogen. Lipids were extracted by the method of Folch. Diacylglycerols were separated by thin-layer chromatography (TLC) according to Roemen and van der Vusse [12]. Authentic lipid class standard was spotted on the outside lanes of the TLC plates to enable localization of sample diacylglycerols. The gel bands corresponding to the standard were scrapped from the plates, transferred into fresh tubes and then transmethylated in 14\% methanolic boron trifluoride (Sigma) at $100^{\circ} \mathrm{C}$ for 10 minutes. The content of resulting fatty acid methyl esters was determined by means of gas-liquid chromatography as previously described in detail [13].

The content of ceramide was determined as described previously in detail [14]. Briefly, tissue was homogenized and lipids were extracted into chloroform. An aliquot of the chloroform phase was subsequently transferred to a fresh tube containing N-palmitoyl-D-erythro-sphingosine (C17 base) (a kind gift of Dr. Z. Szulc, Medical University of South Carolina) as an internal standard. The samples were then subjected to alkaline hydrolysis to deacylate ceramide. Free sphingosine liberated from ceramide was converted to o-phthalaldehyde derivative and analyzed using HPLC system equipped with a fluorescence detector and C18 reversed-phase column (Varian Inc. OmniSpher 5, 4.6×150mm). Since the chloroform extract used for 
ceramide assay contained small amounts of free sphingoid bases, the content of ceramide was corrected for the level of free sphingosine determined in the same sample.

\section{Real-time quantitative PCR}

Total RNA was isolated from the samples using TriReagent (Sigma) according to the manufacturer's instructions. Following RNA purification, DNase treatment (Ambion) was performed to ensure that there was no contaminating genomic DNA. Extracted RNA was solubilized in RNase-free water and stored at $-80^{\circ} \mathrm{C}$ until use. The quality of each RNA sample was verified by running the agarose electrophoresis with ethidium bromide. The RNA was reverse transcribed into cDNA using First Strand cDNA Synthesis Kit (Fermentas) with oligo(dT)18. Oligonucleotide primers were designed using Beacon Designer Software 7.5 (Premier Biosoft). Real-time quantitative PCR was performed with SYBR Green JumpStart Taq ReadyMix (Sigma) using a Bio-Rad Chromo4 system. PCR efficiency was examined by serially diluting the template CDNA, and a melt curve was performed at the end of each reaction to verify PCR product specificity. A sample containing no cDNA was used as a negative control to verify the absence of primer dimers. The results were normalized to $\beta$-actin expression measured in each sample. Primer sequences are available upon request.

\section{Phosphorylation and Western blotting}

Muscle tissues were homogenized in 1:10 (w:v) ice cold lysis buffer (1\% Triton-X100, $20 \mathrm{mM}$ Tris-HCl (pH 7.5), $150 \mathrm{mM} \mathrm{NaCl}$, containing protease and phosphatase inhibitors. Samples were then centrifuged at $10,000 \times g$ for $30 \mathrm{~min}$ at $4^{\circ} \mathrm{C}$, the supernatant was collected and total protein levels were measured by BCA protein assay kit (Pierce). Equal amounts of protein $(50 \mu \mathrm{g})$ were separated by $10 \%$ SDS-PAGE and then transferred on nitrocellulose membranes (BioRad). The membranes were probed with antibodies specific to the proteins analyzed (Abcam). Bounds ware detected after incubation with an alkaline phosphataseconjugated secondary antibody (Sigma). Protein bands were scanned and quantified using a Gel Doc EQ system (Bio-Rad). All samples were also probed with anti- $\beta$-actin antibody to verify equal loading.

For measurement of insulin receptor substrate (IRS) 1 phosphorylation samples were subjected to immunoprecipitation with an anti-IRS-1 antibody (Abcam) followed by incubation with protein A agarose beads (Sigma). The beads were washed extensively, proteins were eluted with Laemmli buffer and then analyzed as described above.

\section{Statistical analysis}

All data are presented as means \pm SD. Statistical comparisons were made by using one-way ANOVA. If overall significance was demonstrated by ANONA, post-hoc multiple comparisons using a Newman-Keuls test were conducted. $\mathrm{P}<0.05$ was considered statistically significant. One, two, or three symbols indicate a significant difference at the $\mathrm{p}<0.05, \mathrm{p}<0.01$, or $\mathrm{p}<0.001$ levels, respectively.

\section{Results}

Animals fed on the high-fat diet were characterized by higher body weight, food consumption and epididymal fat pads weightas compared to the chow-fed rats. Administration of T0901317 did not induce statistically significant changes in the above parameters (Table 1). Plasma insulin, NEFA and triacylglycerols concentrations were increased approximately 2 -fold upon high-fat feeding, however, in the latter case the difference did not reach statistical significance. As expected, LXR agonist induced a further increase in plasma level of triacylglycerols and NEFA, nevertheless, insulin concentration was decreased by the drug (Table 1). In addition, T0901317 administration markedly improved glucose tolerance and decreased insulin tolerance in high-fat fed rats (Fig. 1).

High-fat diet induced a significant decrease in insulin-stimulated 2-deoxy-D-glucose uptake in isolated soleus muscle strips. Although T0901317 tended to ameliorate this effect, the difference did not reach statistical significance. Since basal glucose uptake in this group was slightly lower, we also calculated the delta increase in 2-deoxy-D-glucose uptake (insulin-stimulated - basal), and it was substantially improved upon LXR activation (Fig. 2). 
Table 1. General features and plasma measurements of the experimental animals. The animals were fed for 5 weeks on either standard chow or high fat diet (HFD). The latter group was further divided into two subgroups receiving either vehicle or T0901317 (10 mg/kg/d) throughout the last week of the experiment. The results are means $\pm S D(n=10)$. NEFA - nonesterified fatty acids. ${ }^{*}$ - statistically significant difference vs. standard chow fed group, $\wedge$ - statistically significant difference vs. HFD group. One, two or three symbols indicate a significant difference at the $\mathrm{p}<0.05, \mathrm{p}<0.01$ or $\mathrm{p}<0.001$ levels, respectively

\begin{tabular}{lccc}
\hline & Chow diet & HFD & HFD+T0901317 \\
\hline Initial body weight (g) & $94 \pm 7.9$ & $91.4 \pm 9.6$ & $94.3 \pm 9$ \\
Final body weight (g) & $337 \pm 29$ & $383 \pm 59^{*}$ & $365 \pm 33$ \\
Food consumption (kcal/d) & $99 \pm 13$ & $119 \pm 21^{*}$ & $109 \pm 17$ \\
Epididymal fat pads weight (g) & $3.58 \pm 0.69$ & $6.75 \pm 1.44^{* * *}$ & $6.62 \pm 1.65^{* * *}$ \\
& & & \\
Plasma measurements (fed state) & & & \\
Glucose (mg/dl) & $156 \pm 17$ & $167 \pm 15$ & $147 \pm 22$ \\
Insulin (ng/ml) & $2.94 \pm 1.45$ & $5.26 \pm 1.76^{*}$ & $3.6 \pm 1.65^{\wedge}$ \\
NEFA (nmol/ml) & $183 \pm 32$ & $361 \pm 100^{*}$ & $631 \pm 220^{* * * \wedge \wedge}$ \\
Triacylglycerols (nmol/ml) & $1026 \pm 355$ & $1907 \pm 980$ & $6094 \pm 3113^{* * \wedge}$ \\
\hline
\end{tabular}
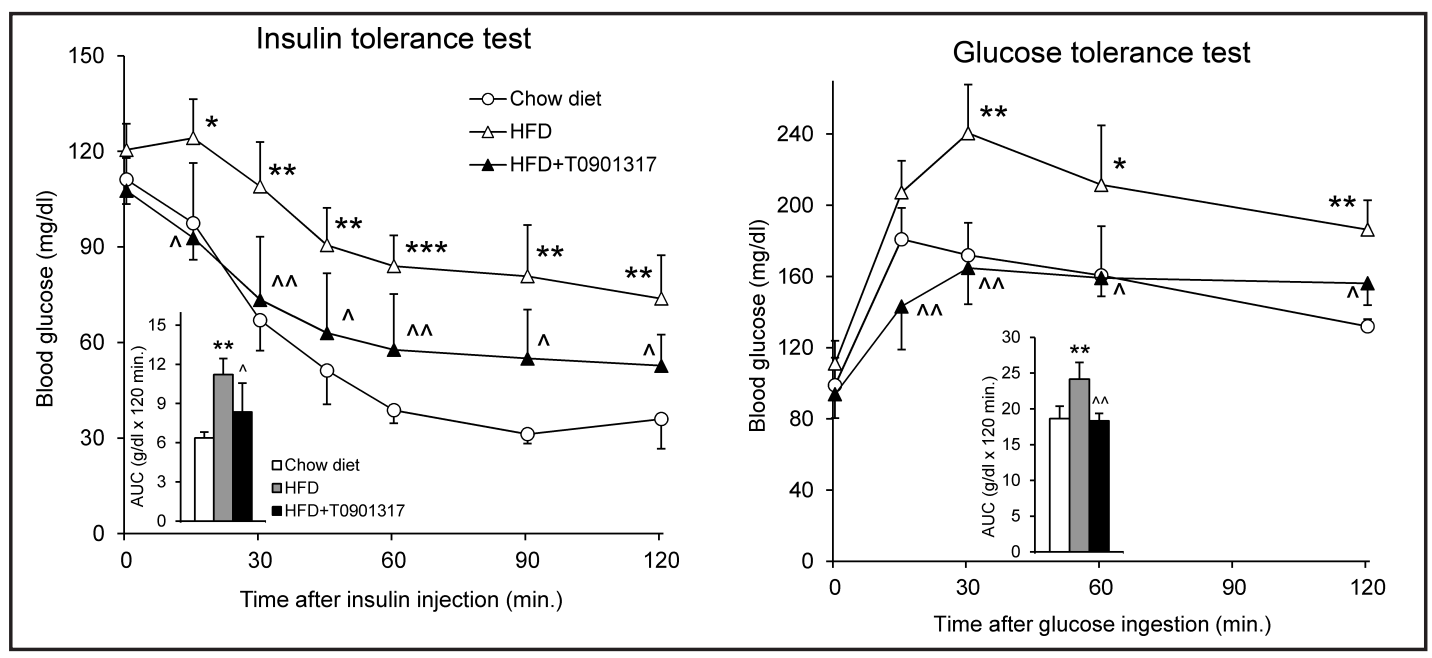

Fig. 1. LXR activation improved whole body insulin sensitivity in high-fat fed rats. The animals were fed for 5 weeks on either standard chow or high fat diet (HFD). The latter group was further divided into two subgroups receiving either vehicle or T0901317 (10 mg/kg/d) throughout the last week of the experiment. Rats were fasted overnight and then subjected to oral glucose tolerance test or intraperitoneal insulin tolerance test. The results are means \pm SD $(n=6)$. AUC - glucose area under the curve. * - statistically significant difference vs. chow diet fed group, ${ }^{\wedge}-$ statistically significant difference vs. HFD group. One, two or three symbols indicate a significant difference at the $\mathrm{p}<0.05, \mathrm{p}<0.01$ or $\mathrm{p}<0.001$ levels, respectively.

Neither high-fat diet nor LXR agonist induced significant differences in total content of IRS-1, Akt/protein kinase B (Akt/PKB) or Akt substrate of $160 \mathrm{kDa}$ (AS160) protein in the examined muscles (data not shown). In general, basal phosphorylation level of muscle Akt/PKB and AS160 was affected by neither high-fat diet nor T0901317. Basal content of phosphorylated IRS-1 protein in the soleus and RG (but not in WG) was moderately decreased upon high-fat feeding, whereas administration of T0901317 induced a marked increase in basal IRS-1 phosphorylation in all muscles examined (Fig. 3). Insulin-stimulated phosphorylation of AS160 was impaired by high-fat diet in each muscle studied. On the other hand, the diet-induced decrease in the level of phosphorylated IRS-1 and Akt/PKB under conditions of insulin stimulation was observed only in the soleus and RG. Administration of T0901317 did not improve insulin-stimulated phosphorylation of IRS-1 or Akt/PKB in 
Fig. 2. LXR activation improved muscle insulinstimulated 2-deoxy-D-glucose uptake in high-fat fed rats. The animals were fed for 5 weeks on either standard chow or high fat diet (HFD). The latter group was further divided into two subgroups receiving either vehicle or T0901317 $(10 \mathrm{mg} / \mathrm{kg} / \mathrm{d})$ throughout the last week of the experiment. Strips of the soleus muscle were then isolated and incubated with ${ }^{3} \mathrm{H}$-2-deoxy-D-glucose in the presence or absence of insulin as described in Materials and Methods. The results are means $\pm S D(n=6) .{ }^{*}$ - statistically significant difference vs. chow diet fed group, $\wedge$ - statistically significant difference vs. HFD group. One or two symbols indicate a significant difference at the $\mathrm{p}<0.05$ or $\mathrm{p}<0.01$ levels, respectively.

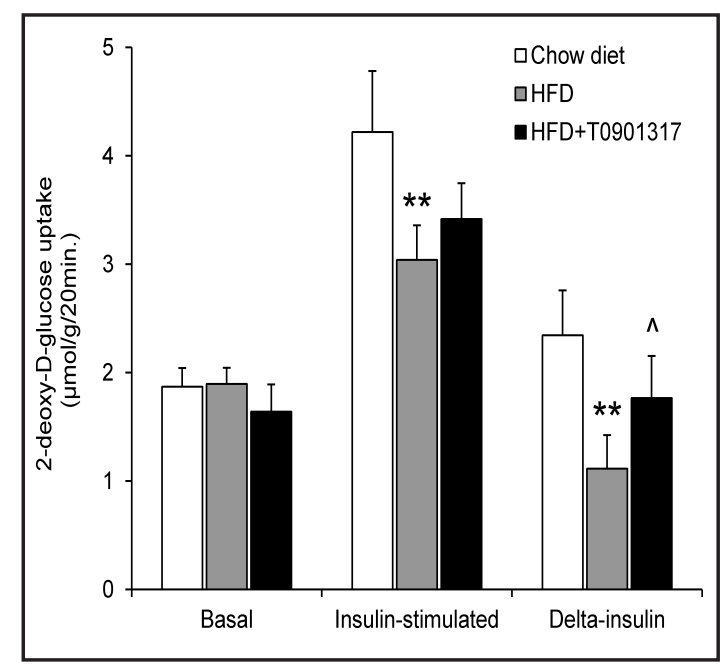

Fig. 3. LXR activation restored insulin-stimulated AS160 phosphorylation in skeletal muscle of high-fat fed rats. The animals were fed for 5 weeks on either standard chow or high fat diet (HFD). The latter group was further divided into two subgroups receiving either vehicle or T0901317 (10 mg/kg/d) throughout the last week of the experiment. Rats were fasted overnight, anaesthetized and muscle samples were taken from one hind limb. A bolus of insulin was then injected into the tail vein and after 4 min muscle samples were excised from the opposite limb. The results are means $\pm \mathrm{SD}(\mathrm{n}=6)$. Data was normalized to the total level of the respective protein determined in the same sample. Akt/PKB - Akt/protein kinase B, AS160 - Akt substrate of $160 \mathrm{kDa}$, IRS-1 - insulin receptor substrate $1 .{ }^{*}$ - statistically significant difference vs. chow diet fed group, ${ }^{\wedge}$ - statistically significant difference vs. HFD group. One, two or three symbols indicate a significant difference at the $p<0.05, p<0.01$ or $\mathrm{p}<0.001$ levels, respectively.

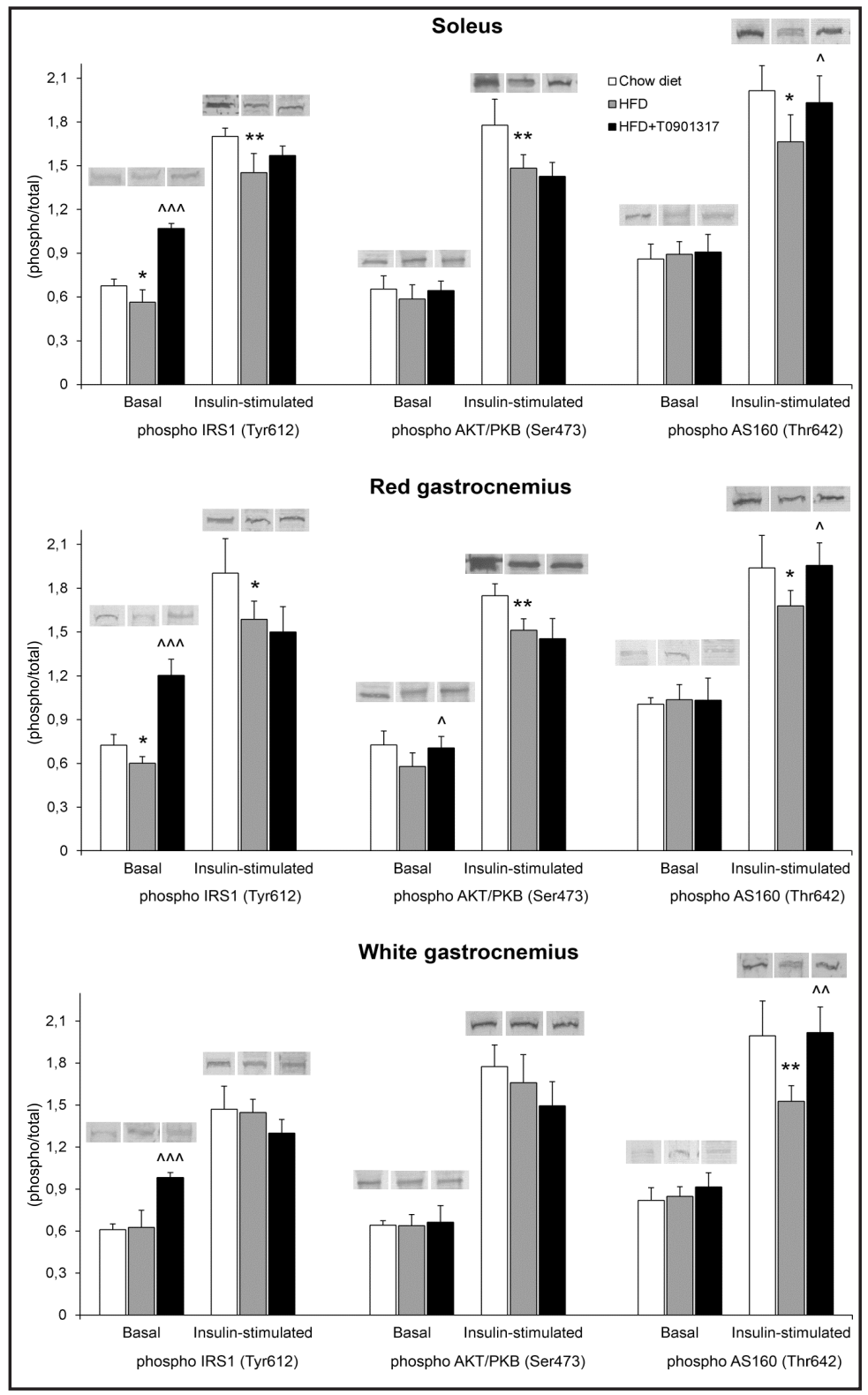


Fig. 4. Effect of high-fat diet and T0901317 on mRNA (italicized) and protein (uppercase) expression of selected genes in skeletal muscle. The animals were fed for 5 weeks on either standard chow or high fat diet (HFD). The latter group was further divided into two subgroups receiving either vehicle or $\mathrm{T} 0901317(10 \mathrm{mg} / \mathrm{kg} / \mathrm{d})$ throughout the last week of the experiment. The results are means $\pm S D(n=6)$. GLUT - glucose transporter, SCD1 - stearoyl-CoA desaturase 1, SREBP-1c - sterol regulatory element binding protein $1 \mathrm{c}$. * - statistically significant difference vs. chow diet fed group, $\wedge$ - statistically significant difference vs. HFD group. One, two or three symbols indicate a significant difference at the $p<0.05, p<0.01$ or $p<0.001$ levels, respectively.

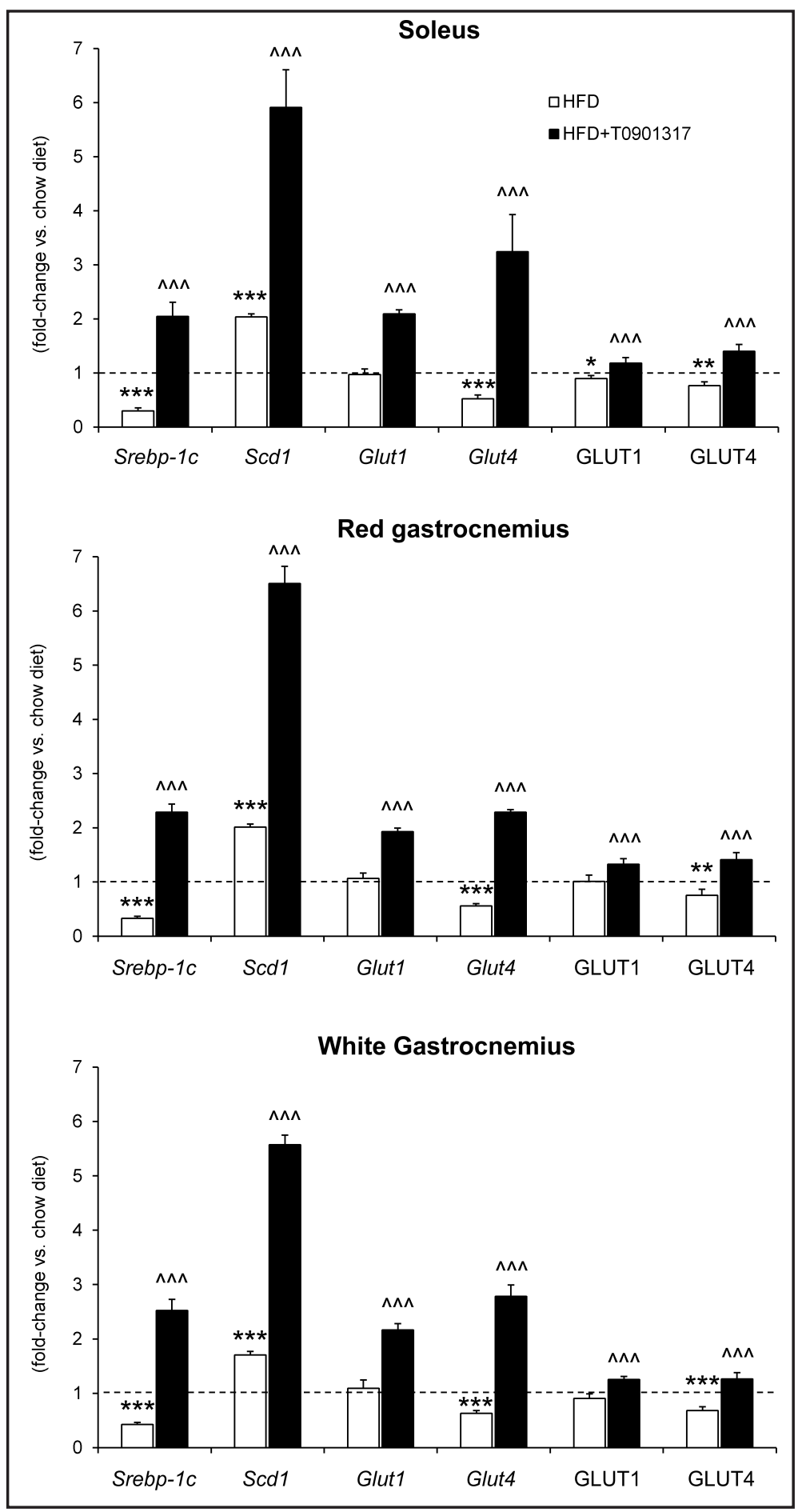

either of the muscles examined. However, the ability of insulin to stimulate phosphorylation of muscle AS160 was completely restored by LXR activation (Fig. 3).

High-fat feeding downregulated SREBP-1c expression and increased stearoyl-CoA desaturase 1 (SCD1) mRNA in all muscles examined. Administration of T0901317 induced a marked increase in expression of these classical LXR target genes indicating efficient receptor activation (Fig. 4). In general, muscle expression of GLUT1 was not affected by highfat feeding, whereas both mRNA and protein levels of GLUT4 were significantly reduced. Administration of T0901317 increased expression of both GLUT isoforms in all muscles 
Fig. 5. Effect of high-fat diet and T0901317 on muscle content of ceramide and diacylglycerol. The animals were fed for 5 weeks on either standard chow or high fat diet (HFD). The latter group was further divided into two subgroups receiving either vehicle or T0901317 (10 mg/kg/d) throughout the last week of the experiment. The results are means \pm SD $(n=10)$. FA - fatty acids, RG - red section of the gastrocnemius muscle, WG - white section of the gastrocnemius muscle. ${ }^{*}$ - statistically significant difference vs. chow diet fed group. One or two symbols indicate a significant difference at the $\mathrm{p}<0.05$ or $\mathrm{p}<0.01$ levels, respectively.

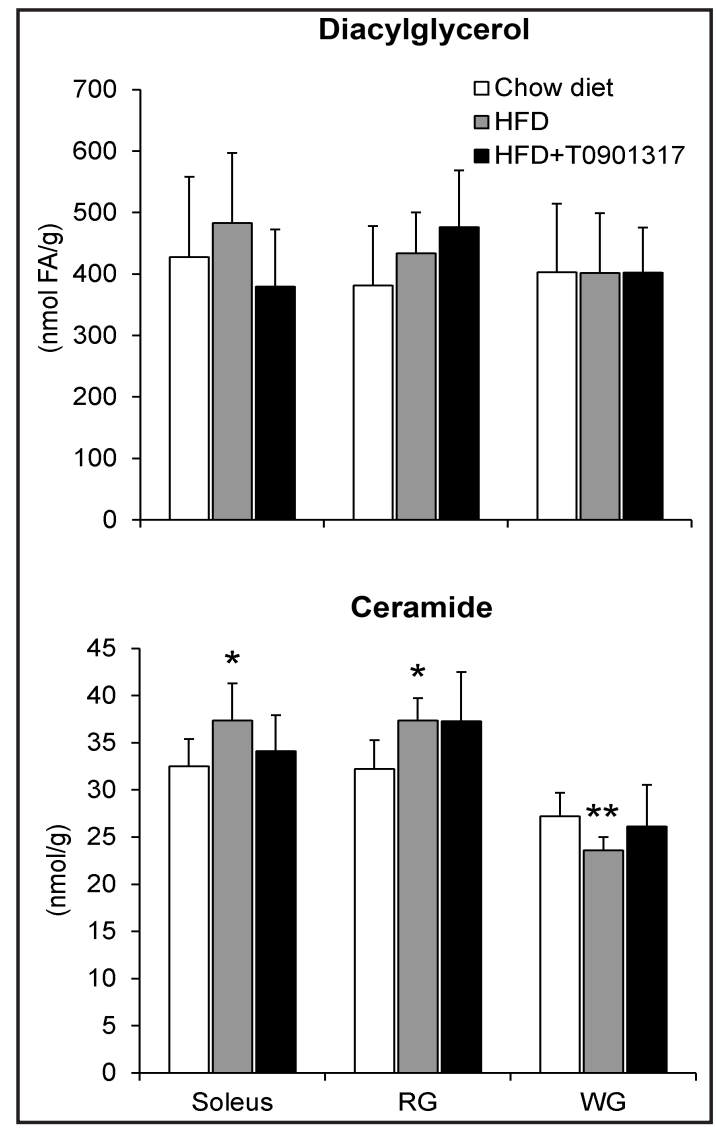

examined. However, this effect was more pronounced for GLUT4, which protein level was not only restored to the baseline, but was significantly greater compared to the lean animals (Fig. 4).

Muscle diacylglycerols content was not affected by either high-fat feeding or T0901317. Rats fed on the high-fat diet were characterized by increased ceramide level in the soleus and RG, whereas in the WG the opposite effect was observed. Administration of LXR agonist did not induce statistically significant changes in ceramide content in any of the muscles examined (Fig. 5).

\section{Discussion}

In the present study administration of synthetic LXR agonist to high-fat fed rats substantially ameliorated whole-body insulin resistance as evidenced by improved glucose tolerance, decreased insulin tolerance, and decreased insulinemia. Similar results were found by other groups in rodent models of genetic and diet-induced insulin resistance [1517].

Although the insulin-sensitizing action of LXR agonists is well documented, it remains unclear whether enhanced insulin-stimulated glucose uptake in peripheral tissues contributes to this effect. Grefhorst et al. [15] found that ob/ob mice treated with GW3965 were characterized by unaltered hepatic glucose production and increased metabolic clearance rate of glucose under hyperinsulinemic conditions. On the contrary, Commerford et al. [17] using euglycemic hyperinsulinemic clamp technique reported that insulinsensitizing effect of GW3965 in high-fat fed rats was a result of suppressed rate of glucose appearance, as peripheral glucose uptake remained stable. In the present study we have found that administration of T0901317 to high-fat fed rats improved the ability of insulin 
to stimulate glucose uptake in isolated soleus muscle. This observation supports the notion that synthetic LXR activators enhance peripheral insulin sensitivity.

Our results are in line with these of Kase et al. [18] who reported that T0901317 increased insulin-stimulated glucose uptake in differentiated myotubes derived from control and type 2 diabetic subjects. In addition, it was shown that insulin-stimulated glucose uptake in insulin-resistant murine and human adipocytes is increased upon incubation with synthetic LXR agonist [19, 20]. However, in contrast to our study Commerford et al. [17] reported that glucose disposal in skeletal muscle during euglycemic hyperinsulinemic clamp was not affected by administration of GW3965 to high-fat fed rats. This discrepancy may be due to the use of different agonists and treatment durations.

T0901317-induced increase in muscle insulin-stimulated glucose uptake that was observed in our study was associated with enhanced insulin signaling at the level of AS160. AS160 is a downstream substrate of Akt/PKB that plays a key role in mediating the translocation of GLUT4 to the plasma membrane in response to insulin [21]. It was found that the ability of insulin to stimulate AS160 phosphorylation is impaired in insulinresistant skeletal muscle, and that this impairment is ameliorated by interventions leading to improved insulin sensitivity [22, 23]. Surprisingly, enhanced phosphorylation of AS160 upon LXR activation, that was found in our study, was not associated with improved Akt/ PKB phosphorylation. Similar observation for rat skeletal muscle was reported also in the study by Bernard et al. [24] where insulin sensitivity was enhanced by amino acid supplementation. We can only speculate as to the mechanism of LXR-induced improvement in muscle insulin-stimulated AS160 phosphorylation. However, our data strongly suggest that this effect is not related to a decrease in the level of lipid mediators of insulin resistance as the intramuscular content of either diacylglycerols or ceramide was not affected by administration of T0901317.

It was previously shown that synthetic LXR agonists ameliorate defects in insulin signaling cascade induced by tumor necrosis factor $\alpha$ or hyperinsulinemia in murine brown adipocytes and human adipocytic cell line, respectively $[19,20]$. However, in contrast to our study, insulin-stimulated Akt/PKB phosphorylation in these cells was markedly improved by T0901317. On the other hand, similarly to our results, Cozzone et al. [25] reported that LXR activation failed to restore Akt/PKB phosphorylation under insulin stimulated conditions in differentiated human myotubes derived from type 2 diabetic subjects. Unfortunately, phosphorylation status of AS160 was not studied in the above reports.

Another effect that likely contributed to increased muscle insulin-stimulated glucose uptake in high-fat fed rats upon LXR activation is elevated GLUT4 expression. In our study, animals treated with T0901317 were characterized by increased mRNA and protein levels of GLUT4 in all examined muscles. A functional LXRE in the promoter region of murine and human GLUT4 gene was identified by Laffitte et al. [16] which indicates that GLUT4 expression is directly regulated by LXRs. Nevertheless, subsequent studies found that LXR activation increases GLUT4 expression in white adipose tissue but not in skeletal muscle of rats and mice [1,15-17], which is in contrast to the results of our study. It should be noted, however, that Dalen et al. [26] reported elevated muscle GLUT4 mRNA level in mice treated with synthetic LXR agonist. Moreover, in differentiated human myotubes GLUT4 expression was markedly upregulated upon incubation with T0901317 [18, 27].

In summary we have found that administration of T0901317 to high-fat fed rats improved whole body insulin sensitivity as well as insulin-stimulated glucose uptake in isolated soleus muscle. In addition, LXR agonist restored muscle GLUT4 expression and insulin-stimulated AS160 phosphorylation that were both reduced upon high-fat feeding. Insulin-sensitizing effect of T0901317 was not related to changes in intramuscular level of lipid mediators of insulin resistance, since neither diacylglycerols nor ceramide content was affected by the drug. We conclude that improvement in muscle insulin sensitivity is one of the mechanisms underlying the antidiabetic action of LXR activators. 


\section{Acknowledgements}

This work was supported by the Polish Ministry of Science and Higher Education (grant no. N401 134 31/2926) and by the Medical University of Bialystok (grants no. 134-18713 and 134-18714). The authors have no conflicts of interest to declare.

\section{References}

1 Ulven SM, Dalen KT, Gustafsson JA, Nebb HI: LXR is crucial in lipid metabolism. Prostaglandins Leukot Essent Fatty Acids 2005;73:59-63. Baranowski M: Biological role of liver X receptors. J Physiol Pharmacol 2008;59:S31-55. Wojcicka G, Jamroz-Wisniewska A, Horoszewicz K, Beltowski J: Liver X receptors (LXRs). Part I: structure, function, regulation of activity, and role in lipid metabolism. Postepy Hig Med Dosw (Online) 2007;61:736759.

4 Bjorkhem I, Meaney S, Diczfalusy U: Oxysterols in human circulation: which role do they have? Curr Opin Lipidol 2002;13:247-253.

5 Schultz JR, Tu H, Luk A, Repa JJ, Medina JC, Li L, Schwendner S, Wang S, Thoolen M, Mangelsdorf DJ, Lustig KD, Shan B: Role of LXRs in control of lipogenesis. Genes Dev 2000;14:2831-2838.

6 Joseph SB, McKilligin E, Pei L, Watson MA, Collins AR, Laffitte BA, Chen M, Noh G, Goodman J, Hagger GN, Tran J, Tippin TK, Wang X, Lusis AJ, Hsueh WA, Law RE, Collins JL, Willson TM, Tontonoz P: Synthetic LXR ligand inhibits the development of atherosclerosis in mice. Proc Natl Acad Sci USA 2002;99:7604-7609.

-7 Terasaka N, Hiroshima A, Koieyama T, Ubukata N, Morikawa Y, Nakai D, Inaba T: T-0901317, a synthetic liver $\mathrm{X}$ receptor ligand, inhibits development of atherosclerosis in LDL receptor-deficient mice. FEBS Lett 2003;536:6-11.

8 Faulds MH, Zhao C, Dahlman-Wright K: Molecular biology and functional genomics of liver X receptors (LXR) in relationship to metabolic diseases. Curr Opin Pharmacol 2010;10:692-697.

-9 Abdallah BM, Beck-Nielsen H, Gaster M: Increased expression of 11beta-hydroxysteroid dehydrogenase type 1 in type 2 diabetic myotubes. Eur J Clin Invest 2005;35:627-634.

10 Zhang Y, Mangelsdorf DJ: LuXuRies of lipid homeostasis: the unity of nuclear hormone receptors, transcription regulation, and cholesterol sensing. Mol Interv 2002;2:78-87.

11 Hansen PA, Gulve EA, Holloszy JO: Suitability of 2-deoxyglucose for in vitro measurement of glucose transport activity in skeletal muscle. J Appl Physiol 1994;76:979-985.

12 Roemen TH, van der Vusse GJ: Application of silica gel column chromatography in the assessment of nonesterified fatty acids and phosphoglycerides in myocardial tissue. J Chromatogr 1985;344:304-308.

13 Nawrocki A, Gorski J: Effect of plasma free fatty acid concentration on the content and composition of the free fatty acid fraction in rat skeletal muscles. Horm Metab Res 2004;36:601-606.

14 Baranowski M, Zabielski P, Blachnio A, Gorski J: Effect of exercise duration on ceramide metabolism in the rat heart. Acta Physiol (Oxf) 2008;192:519-529.

15 Grefhorst A, van Dijk TH, Hammer A, van der Sluijs FH, Havinga R, Havekes LM, Romijn JA, Groot PH, Reijngoud DJ, Kuipers F: Differential effects of pharmacological liver X receptor activation on hepatic and peripheral insulin sensitivity in lean and ob/ob mice. Am J Physiol Endocrinol Metab 2005;289:E829-E838.

16 Laffitte BA, Chao LC, Li J, Walczak R, Hummasti S, Joseph SB, Castrillo A, Wilpitz DC, Mangelsdorf DJ, Collins JL, Saez E, Tontonoz P: Activation of liver X receptor improves glucose tolerance through coordinate regulation of glucose metabolism in liver and adipose tissue. Proc Natl Acad Sci USA 2003;100:5419-5424.

17 Commerford SR, Vargas L, Dorfman SE, Mitro N, Rocheford EC, Mak PA, Li X, Kennedy P, Mullarkey TL, Saez E: Dissection of the insulin-sensitizing effect of liver X receptor ligands. Mol Endocrinol 2007;21:30023012.

18 Kase ET, Wensaas AJ, Aas V, Hojlund K, Levin K, Thoresen GH, Beck-Nielsen H, Rustan AC, Gaster M: Skeletal muscle lipid accumulation in type 2 diabetes may involve the liver $\mathrm{X}$ receptor pathway. Diabetes 2005;54:1108-1115.

19 Fernandez-Veledo S, Nieto-Vazquez I, Rondinone CM, Lorenzo M: Liver X receptor agonists ameliorate TNFalpha-induced insulin resistance in murine brown adipocytes by downregulating protein tyrosine phosphatase-1B gene expression. Diabetologia 2006;49:3038-3048. 


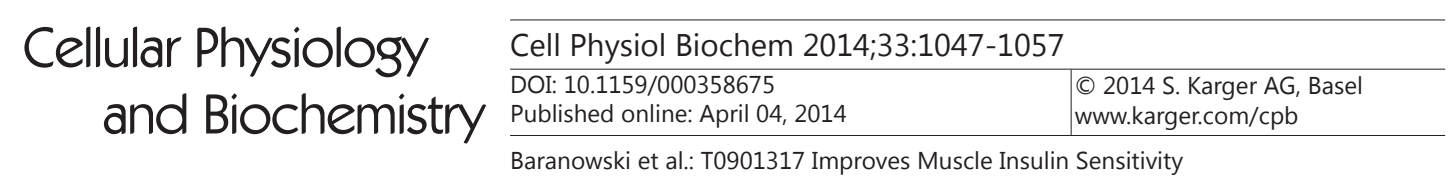

20 Fernandez-Veledo S, Nieto-Vazquez I, de Castro J, Ramos MP, Bruderlein S, Moller P, Lorenzo M: Hyperinsulinemia induces insulin resistance on glucose and lipid metabolism in a human adipocytic cell line: paracrine interaction with myocytes. J Clin Endocrinol Metab 2008;93:2866-2876.

-21 Gomez-Garre D, Herraiz M, Gonzalez-Rubio ML, Bernal R, Aragoncillo P, Carbonell A, Rufilanchas JJ, Fernandez-Cruz A: Activation of peroxisome proliferator-activated receptor-alpha and -gamma in auricular tissue from heart failure patients. Eur J Heart Fail 2006;8:154-161.

-22 Jazet IM, Schaart G, Gastaldelli A, Ferrannini E, Hesselink MK, Schrauwen P, Romijn JA, Maassen JA, Pijl H, Ouwens DM, Meinders AE: Loss of $50 \%$ of excess weight using a very low energy diet improves insulinstimulated glucose disposal and skeletal muscle insulin signalling in obese insulin-treated type 2 diabetic patients. Diabetologia 2008;51:309-319.

23 Hojlund K, Glintborg D, Andersen NR, Birk JB, Treebak JT, Frosig C, Beck-Nielsen H, Wojtaszewski JF: Impaired insulin-stimulated phosphorylation of Akt and AS160 in skeletal muscle of women with polycystic ovary syndrome is reversed by pioglitazone treatment. Diabetes 2008;57:357-366.

24 Bernard JR, Liao YH, Hara D, Ding Z, Chen CY, Nelson JL, Ivy JL: An amino acid mixture improves glucose tolerance and insulin signaling in Sprague-Dawley rats. Am J Physiol Endocrinol Metab 2011;300:E752-E760.

25 Cozzone D, Debard C, Dif N, Ricard N, Disse E, Vouillarmet J, Rabasa-Lhoret R, Laville M, Pruneau D, Rieusset J, Lefai E, Vidal H: Activation of liver X receptors promotes lipid accumulation but does not alter insulin action in human skeletal muscle cells. Diabetologia 2006;49:990-999.

-26 Dalen KT, Ulven SM, Bamberg K, Gustafsson JA, Nebb HI: Expression of the insulin-responsive glucose transporter GLUT4 in adipocytes is dependent on liver X receptor alpha. J Biol Chem 2003;278:4828348291.

27 Kase ET, Thoresen GH, Westerlund S, Hojlund K, Rustan AC, Gaster M: Liver X receptor antagonist reduces lipid formation and increases glucose metabolism in myotubes from lean, obese and type 2 diabetic individuals. Diabetologia 2007;50:2171-2180. 